\title{
STORIA DEI FILOSOFI E TEOLOGIA DELLA STORIA IN AGOSTINO E NELL'ETÀ MEDIEVALE'
}

Gregorio Piaia“

SÍNTESE - A costumeira afirmação de que a historiografia nasce com o iluminismo deixa, por vezes, de lado o fato de que no passado também se pensou em categorias históricas e fez-se uma história dos filósofos e uma teologia da história, com os recursos e técnicas que nem sempre são bem conhecidas.
ABSTRACT - The usual affirmation that the historiography has its origin in the illuminism doesn't consider the fact that also in past times historical categories were taken in consideration and forgets that also a history of the philosophers as well as a theology of the history were elaborated with the resources and techniques which usually are almost unknown.

Se la nozione di "storicità" del sapere, e in particolare del sapere filosofico, appare ovvia ai nostri occhi smaliziati da oltre due secoli di storicismo, non altrettanto chiara ed evidente è la genesi remota del processo che ha portato alla elaborazione di nessi categoriali come "storia della sapienza": o "storia della verità", archetipi di quella che siamo soliti chiamare - sia pure in tutt'altra prospettiva teorica - "storia della filosofia". La domanda che ci poniamo al riguardo è la seguente: con quali forme e modalità la cultura medievale ha 'letto' e ricostruito il passato della filosofia, e ne ha quindi in qualche modo riconosciuto la "storicità"? Impossibile qui prescindere da un richiamo a s. Agostino e alla sua grandiosa visione della storia come historia salutis, all'interno della quale un ruolo di primo piano è svolto da quella che chiameremo "storia dei filosofi", per distinguerla dal moderno concetto di "storia della filosofia", profondamente segnato da una 'teologia laica' della cultura, qual è quella illuministica e hegeliana.

Il presente articolo è una sintesi del ciclo di lezioni tenuto nel settembre 1996 alla PUCRS (Curso de Pós-Graduação em Fillosofia). Per una più ampia trattazione del tema rinviamo alla prima parte del nostro volume Vestigia philosophorum. Il medioevo e la storiografia filosofica, Rimini, Maggioli, 1983, pp. 13-159.

Università di Padova. 
Giova soffermarsi, a questo proposito, sui primi capitoli di quell'VIII libro del De civitate Dei in cui Agostino opera una significativa mediazione fra le nuove istanze della dottrina cristiana e la tradizionale letteratura dossograficodiadochistica, ereditata dal mondo greco. E una mediazione critica, giacché il 'racconto' che ha per oggetto gli antichi filosofi è guidato dal giudizio sulle loro posizioni speculative, che sono commisurate a un concetto ben definito di "filosofia" e di "verità", in grado di fornire un senso alla estrinseca successione (diadochè) di filosofi e di dottrine. Il taglio teorico che Agostino adotta in questo suo excursus è illustrato nel capitolo introduttivo: se la sapientia si identifica con Dio, vero filosofo è colui che ama Dio (amator Dei); ma poichè non tutti i filosofi, malgrado l'epiteto di cui si fregiano, sono amanti della vera sapienza, occorre distinguere e trascegliere. Tale "delimitazione del campo" - così la chiameremmo oggi - è condotta su due versanti, in quanto concerne sia la materia d'indagine sia le personalità e le scuole filosofiche. I vescovo d'Ippona intende infatti limitarsi alla teologia naturale, e di tale argomento vuole discutere non con tutti i filosofi indistintamente, ma solo con i "Platonici", i quali, pur riconoscendo l'esistenza di Dio e di un Dio provvidente, promuovono nel contempo il culto di molti dèi, generati e ispirati da quell'unico Dio. Rispetto allo stesso Varrone, che è la grande fonte nella trattazione dei diversi tipi di teologia, i filosofi "platonici" segnano un indubbio progresso, poichè si avvicinano maggiormente alla verità, ossia alla dottrina della natura incorporea di Dio e dell'anima. Al centro dell'interesse di Agostino sta dunque la teologia platonica, ed è a questo interesse eminentemente dottrinale che viene finalizzata la trattazione storica: prima di discutere su Platone, egli menzionerà quanti hanno 'preceduto' il filosofo greco su questo arduo terreno d'indagine ("...prius illos commemorans, qui eum in eodem genere litterarum tempore praecesserunt”).'

Entro questa cornice Agostino dispone il materiale dossografico, ripartito fra i due tradizionali "philosophorum genera", la scuola Italica e la scuola Ionica, per poi passare a Socrate e ai suoi discepoli, fra i quali primeggia Platone (cap. ii-iv). Tale rassegna lascia intravvedere una visione evolutiva della storia filosofica, giacché il vescovo di Ippona non intende solo presentare una genealogia estrinseca di filosofi da Talete a Platone, ma vuole cogliere nelle dottrine di questi 'predecessori' i segni delle più mature dottrine platoniche, che fungono da termine di paragone. Quella che in qualche modo egli propone è una rilettura del pensiero antico all'interno degli schemi consacrati dalla tradizione storiografica. Ed ecco allora che in Talete ed Anassimandro viene denunciata l'assenza di qualsiasi "mente divina" nell'opera della natura, mentre fu Anassagora che pose un "animo divino" all'origine del mondo ("Anaxagoras vero [...] harum rerum omnium, quas videmus, effectorem divinum animum sensit"). La svolta in senso morale impressa da Socrate all'indagine filosofica viene ricondotta all'affermarsi di una più profonda esigenza teologica, per cui la ricerca delle "cause prime e somme delle cose" si orienta verso la "volontà di un Dio unico e sommo", anche se poi - nota Agostino

AUG., Civ. 8, 1 (Corpus Christianorum. Series Latina [=CCL] 47, pp. 216-217). 
- non risulta chiaramente che cosa sia per il filosofo ateniese quel sommo bene da cui dipende la felicità?.

Questo modo di procedere non è certo nuovo, poiché già Aristotele nel libro I della Metafisica aveva esaltato il "senno" di Anassagora, rispetto al quale "i predecessori sembrarono gente che parla alla ventura", ed anche Cicerone, ad es., nel De natura deorum $(1,11,26)$ aveva rilevato che il filosofo di Clazomene era stato il primo a porre una mente infinita come regola ordinatrice di tutte le cose. Ma il dato significativo, in questo caso, non è tanto la novità del procedimento, quanto la ripresa di un approccio ai pensatori antichi animato da una tensione dottrinale che non si ritrova nei testi dei dossografi e dello stesso Cicerone, impiegati da Agostino come fonte. D'altro canto l'impostazione speculativa del discorso storico in Aristotele e in Agostino avviene secondo coordinate diverse, che rinviano a differenti livelli di "storicità". Nel libro I della Metafisica l'operazione storicofilosofica assume la veste di un sistematico status quaestionis elaborato in base alle categorie e alle istanze della filosofia propria di Aristotele, anche se questa dovrebbe rispecchiare fedelmente - dato il tipico realismo aristotelico - la verità in sè delle strutture ontologiche, da sempre esistenti e in sè perfette: una verita unica ed uniforme, che si manifesta nella dottrina delle quattro cause prime e che, secondo una "concezione circolare della storia", fu "ciclicamente scoperta e poi perduta dagli uomini, che tuttavia di essa conservano sempre qualche reliquia, finchè non la riscoprono del tutto"; ${ }^{4}$ ed Aristotele è profondamente convinto di essere il protagonista di tale definitiva scoperta.

Per Agostino, invece, si tratta di rileggere in modo unitario una tradizione filosofica multiforme e frastagliata, piena di luci e di ombre, e di attribuirle un senso; e ciò sulla base di un criterio ancorato alla Sapienza stessa di Dio, che ha creato il mondo, rivelandosi poi nella storia e nell'uomo, e facendosi essa stessa uomo-nella-storia. Sullo sfondo vi è la prospettiva della historia salutis, che costituisce una frattura netta con la concezione greca del divenire storico. Si pensi, ad es., al ruolo che nell'esposizione del pensiero platonico viene assunto dalla tripartizione della filosofia in fisica, etica e logica. Questo schema, che a partire dall'età ellenistica era di uso corrente nelle scuole di filosofia, viene qui vivificato e trasfigurato alla luce della struttura trinitaria dell'uomo, imago della Trinità divina, e in tale nuova veste è ripetutamente applicato e costituisce anzi una sorta di Leitmotiv.

L'impiego del materiale storico-filosofico non si esaurisce nella prospettiva della 'introduzione' ai "Platonici", ma avviene anche nell'ambito di un confronto critico. Che bisogno c'è di esaminare gli altri filosofi - si chiede con enfasi Agostino all'inizio del cap. 5 - se già Platone ha definito il sapiente come colui che imita, conosce ed ama il Dio unico e vero? Questo riconoscimento dà luogo ad un confronto costruito con retorica sapiente, in cui la successione cronologica dei filosofi che hanno preceduto Platone è soppiantata dalla classificazione delle dot-

\footnotetext{
AUG., Civ. 8, 3 (CCL 47, pp. 218-219).

ARISTOT., Metaph. I, 3, 984 b 15-19 (tr. it. di G. Reale, Napoli, Loffredo, 1968, p. 113).

Ibi, p. 151.

Cfr., ad es., AUG., Civ. 8, 4 (CCL 47, pp. 220-221).
} 
trine sulla base del loro grado di vicinanza alla verità. Così, di fronte ai "Platonici" debbono cedere non soltanto la teologia "favolosa" e "civile", le interpretazioni di Varrone, le rivelazioni di Numa Pompilio ed Alessandro Magno, ma anche i filosofi a pieno titolo come Talete, Anassimandro, gli Stoici ed Epicuro, che posero principi corporei all'origine di tutte le cose. Ad un gradino superiore sono collocati quei filosofi (i Pitagorici?) che concepirono Dio della medesima natura dell'anima: ma anche costoro sono superati dai Platonici, i quali posero la natura immutabile di Dio al di sopra della mutabile anima umana. Lo schema storico della diadochè si dissolve cos 1 in uno schema teoretico, mentre all'idea di uno sviluppo progressivo culminante nei platonici subentra il senso vivo del distacco tra questi filosofi e tutti gli altri. ${ }^{6}$

Al di là di tale dialettica permane tuttavia una concezione unitaria del pensiero umano, che si riflette nell'ampio periodo che, da solo, costituisce il cap. 9. Qui la prospettiva si allarga e intorno al concetto tipicamente agostiniano di un "Dio sommo e vero [...] creatore di tutte le cose, luce delle nostre conoscenze, bene a cui tendono le nostre azioni", si raccoglie un vasto consenso di filosofi: dai Platonici ai più eminenti fra gli Ionici e gl'Italici, sino ai "sapienti o filosofi di altre nazioni, Libici, Atlantici, Egizi, Indiani, Persiani, Caldei, Sciti, Galli, Spagnoli”.' Un accordo sostanziale sul modo di concepire la divinità unisce dunque le sette greche e le nazioni "barbare", e avvicina tutti questi sapienti alla verità del cristianesimo; anche se poi quest'ultimo (è l'argomento del successivo cap. 10) si rivela in ogni caso superiore alla filosofia dei gentili ed ha nei suoi confronti quell'atteggiamento ambivalente di diffidenza/apertura che l'apostolo Paolo aveva emblematicamente espresso nelle sue lettere ai Romani $(1,18-23)$ e ai Colossesi $(2,8)$. Il tema della "filosofia barbarica", che era già stato oggetto di discussione nel proemio delle Vite di Diogene Laerzio ed era stato toccato da autori cristiani come Cirillo d'Alessandria, Eusebio e Clemente Alessandrino, viene così inserito in modo autorevole nel circuito storico dell'umana sapienza.

Frutto della dossografia ellenistico-romana e della più matura riflessione patristica, questa trattazione di Agostino si pone a sua volta all'inizio di una lunga e ricca tradizione storiografica. Infatti l'autorità indiscussa del vescovo di Ippona e l'oggettiva scarsità di fonti più dirette fecero sì che questo ed altri excursus storico-filosofici del De civitate assurgessero al rango di fonte corrente o addirittura esclusiva sui pensatori antichi: il tutto nel quadro di una teologia della storia e della cultura che offriva un sicuro sistema di riferimento sul piano insieme intellettuale e spirituale. Seguire l'evolversi di questa tradizione storiografica e il suo arricchirsi e variare a contatto di altre tradizioni consente di gettare luce su quel particolare aspetto delia coscienza storica medievale rappresentato dalla storia della sapienza e dalla storia dei filosofi. Ci limitiamo in questa sede a segnalare alcuni fra i momenti più rappresentativi di tale processo.

Iniziamo dal celebre Chronicon sive Historia de duabus civitatibus di Ottone di Frisinga (+ 1158), di impianto tipicamente agostiniano, ove però possiamo già cogliere i primi indizi della grande svolta impressa alla cultura latino-medievale

AUG., Civ. 8, 5 (CCL 47, pp. 219-220).

AUG., Civ. 8, 9 (CCL 47, pp. 225-226). 
dalla riscoperta di Aristotele. Il capitolo viii del libro II, che porta il titolo "De philosophis praecipue Pitagora, Socrate, Platone, Aristotile", è in realtà quasi interamente dedicato a questi due ultimi pensatori. Dopo aver accennato alla successiva comparsa dei poeti-teologi, dei sapienti e dei philosophi veri e propri, Ottone menziona Pitagora e la sua scoperta degli intervaili musicali, e poi Socrate, presentato come l' "educatore" di Platone ed Aristotele. Fedele alla sua ispirazione agostiniana, sin qui il vescovo di Frisinga non ha fatto che ricalcare le orme del vescovo di Ippona, ma nelle righe seguenti il discorso imbocca un cammino del tutto nuovo. Infatti, se il ritratto di Platone è conforme alla tradizione agostiniana, diverso e ben più ricco è il profilo di Aristotele, che Agostino s'era limitato a definire "uomo di eletto ingegno, inferiore a Platone per lo stile, ma superiore a molti altri (Civ. 8, 12). Memore dei suoi studi di filosofia a Parigi, Ottone dà grande risalto all'Aristotele logico, fornendo il titolo degli scritti che compongono l'Organon e precisando, con brevità ma pertinenza, il contenuto di ognuno. Inoltre egli mostra di aver colto la funzione che la logica aristotelica svolge nel "perfetto filosofo"; essa non serve solo a conoscere la verità, ma ad evitare l'errore:

"Quorum alter [= Platone] de potentia, sapientia, bonitate creatoris ac genitura mundi creationeve hominis tam luculenter, tam sapienter, tam vicine veritati disputat, ut ob hoc a quibusdam ex nostris Hieremiam in Aegypto audisse, et ab eo de fide nostra imbutus fuisse credatur. Alter [= Aristotele] logicam in sex libros id est praedicamenta, peri ermenias, priora analetica, topica, posteriora analetica, elencos distinxit. Quorum primus de simplicibus terminis, secundus de propositionibus, tertius de complexione propositionum utili ad syllogizandum iudicium purgans et instruens, quartus de methodis id est via syllogizandi, quintus de demonstrationis necessitate, sextus de cautela sophisticarum fallaciarum docet. Ut ita perfectum philosophum non solum ad cognoscendae veritatis, sed ad vitandae falsitatis scientiam perfecte informet". ${ }^{\text {. }}$

A testimonianza del posto occupato da Aristotele quale "princeps" e "inventor" della logica e, in particolare, quale iniziatore di un rigoroso metodo sillogistico, Ottone riporta poi per esteso due brani tratti dalla conclusione degli Elenchi sofistici. Inoltre egli fa presente che anche prima di Aristotele si usava il procedimento sillogistico, ma in modo casuale e con esiti incerti, e a riprova di ciò riproduce un presunto sillogismo di Platone (citato da Boezio nel suo commento al De interpretatione) che lo Stagirita avrebbe confutato negli Analitici primi. ${ }^{9}$

Questa digressione sulla logica aristotelica è decisamente estranea al sentire di Agostino, il quale nelle Confessioni $(4,16)$ dichiara di aver letto in gioventù le Categorie, che però non gli furono di alcun giovamento e lo indussero anzi ad un'errata concezione del divino. Dopo tale digressione Ottone riprende il discorso su Platone, che aveva lasciato in sospeso. Così egli osserva che il filosofo ateniese visse in realtà dopo il profeta Geremia (cfr. Civ. 8, 11) e ripropone quindi l'interpretazione agostiniana di Platone e dei "Platonici": muovendo dalle cose visibili, essi riuscirono a cogliere con le forze dell'ingegno umano le realtà invisibili, fermandosi solo davanti alle verità che sono essenziali per l'eterna salvezza e

OTTONIS EP. FRISINGENSIS Chronicon, 2, 8 (MGH SS 20, p. 147).

Ibidem. 
che ci sono state rivelate dalla grazia divina. Ė per questo - rileva Ottone - che Agostino affermava di aver trovato in Platone la dottrina del Verbo creatore, ma non quella del Verbo incarnato (cfr. Conf. 7, 9). ${ }^{10}$

Nel prologo al libro III del Chronicon a questi puntuali richiami all'interpretazione agostiniana del platonismo corrisponde - sul piano dell'interpretazione generale del corso della storia umana - la ripresa del tema patristico della "preparazione" dell'avvento di Cristo. All'interno del grande disegno provvidenziale, che dà senso e direzione al movimento della storia, la filosofia dei Greci svolge un ruolo preciso, collocandosi tra la vicenda del popolo d'Israele e il sorgere dell'impero di Roma: insieme con la "virtus Romanorum", la "sapientia philosophorum" ha contribuito a rendere i tempi maturi per la comparsa del Salvatore e l'instaurazione di una nuova legge. La funzione degli antichi filosofi nell'economia salvifica ottiene cosi, in una sede storiografica qualificata, un riconoscimento e una piena legittimazione."

Ma tomiamo alla digressione aristotelica inserita da Ottone entro il quadro agostiniano, indice di un mutato atteggiamento verso i filosofi dell'antichità. Nel Chronicon Aristotele non è soltanto un discepolo del grande Platone, né figura quale uno dei "filosofi gentili" ispiratori di eresie (come in Isidoro di Siviglia, Etym. 8,6), ma è il maestro di un metodo dimostrativo indispensabile al "perfectus philosophus". Per tale via i legami con la filosofia antica vengono riannodati ed estesi al di là del pensiero di Platone, il più 'cristiano' fra i gentili, ed assumono un carattere schiettamente razionale. Si pongono così le premesse - anche sul piano storiografico - per il superamento dello schema dei philosophi gentium, entro cui la tradizione patristica aveva racchiuso e bloccato la prospettiva storico-filosofica. Aristotele si avvia ad essere riconosciuto come l'espressione più alta dell'umana ragione (il Philosophus per antonomasia) e in quanto tale egli è valido in ogni tempo. Per Ottone, che aveva contribuito attivamente al rinnovamento filosofico del secolo XII, introducendo in Germania i testi della logica nova, la philosophia non è soltanto un episodio circoscritto al mondo pagano, da Talete ad Epicuro e ai Gimnosofisti, ma esiste ed è praticata anche ai suoi giorni, in un rapporto di continuità con il passato pre-cristiano. La via verso una storia generale del pensiero, che si allarghi ai filosofi più recenti e giunga "usque ad tempus nostrum", è oramai aperta.

Questa prospettiva appare compiutamente raggiunta, a distanza di poco più di un secolo, nella Summa philosophiae dello pseudo Grossatesta, composta negli anni 1260-1270. Il primo dei 19 trattati in cui è ripartita questa trattazione sistematica del pensiero filosofico, teologico e scientifico presenta anche un breve schizzo storico-filosofico, imperniato su una divisione in quattro grandi periodi che coprono quasi l'intera storia dell'umanità. I primi due periodi (dal tempo di Noè sino al regno di Ciro il Grande, e da questo all'età di Alessandro Magno) corrispondono alla periodizzazione in uso nelle storie universali, di cui la 'storia dei filosofi' viene a rappresentare, per così dire, una sezione. Il terzo periodo è quello dei "filosofi romani", siano essi di lingua greca o latina, e va dalla conquista ro-

11 Ibidem.

$"$ OTTON. Chron. 3, Prol. (MGH SS 20, p. 170). 
mana della Grecia e dell'Oriente fino al tempo degli Arabi. Il più interessante è il quarto ed ultimo periodo, cui è riservato un capitolo dal titolo "De philosophis famosis arabicis vel hispanis et aliis eis vel contemporaneis vel succedentibus etiam latinis". L'inizio di tale periodo è fissato al tempo dell'imperatore bizantino Eraclio ed è ricondotto - al pari dei precedenti - a un grande evento storico, che modificò profondamente non solo la geografia politica ma anche quelia culturale: l'avanzata dell'Islam ad oriente e, ad occidente, in Ispagna e in Gallia. Seguendo la tripartizione religiosa che è ancor oggi tenuta presente dagli storici della filosofia medievale, l'autore della Summa menziona i più celebri filosofi musulmani, alcuni cristiani e due ebrei (Mosè Maimonide e il convertito Pietro Alfonso, che si chiamava pure lui Mosè). Quanto ai "filosofi cristiani", si tratta in realtà di traduttori (Platone Tiburtino, Costa ben Luca, Domenico Gundisalvi, Costantino Africano) e di autori di opere scientifiche, come il medico bizantino Teofilo Protospatario (sec. VII), di cui erano noti in occidente il De pulsibus e il De urinis, e Macer Floridus, pseudonimo dell'autore del poema De viribus seu de virtutibus herbarum. Sono tutti personaggi che giungono cronologicamente sino al XII secolo. L'autore della Summa precisa tuttavia che vi sono parecchi altri eccellenti filosofi, menzionandone alcuni: un "Johannes Peripateticus", Alfredo di Sareshel e due "moderniores", ossia contemporanei, il francescano Alessandro di Hales (+ 1245) e il domenicano Alberto Magno. ${ }^{12}$

E soltanto una sequela di nomi che ci viene offerta in queste pagine introduttive della Summa philosophiae; eppure questa nuda successione di nomi, distribuiti nei quattro periodi, è indice dell'avvenuta assimilazione storiografica del pensiero più remoto, risalente ai figli di Noè, e del pensiero più 'moderno', oltre che, naturalmente, di quello greco-romano. In una parola, siamo di fronte ad uno dei primi e più significativi modelli di storicizzazione integrale della filosofia, intesa come attività che accompagna ogni epoca dell'umano cammino. Questa visione unitaria ha evidenti implicazioni teologiche, tant'è vero che nella Summa philosophiae viene adottato anche un altro schema di periodizzazione, che s'ispira a un criterio apologetico-speculativo anziché strettamente storico. Nel capitolo "Quod omnes antiqui philosophi usque ad tempora Theodosii magni in aestimatione et cultu divinitatis enormiter erraverunt et in aliqua opinione singulares extiterunt", la storia dei filosofi risulta infatti divisa in due soli, grandi periodi, fra i quali è posto un netto salto di qualità. Sino alla fine del quarto secolo dopo Cristo tutti gli antichi filosofi sbagliarono il modo d'intendere e adorare la divinità, in quanto il riconoscimento di un Dio supremo (Giove) si accompagnò alla credenza in una molteplicità di dei, fossero essi fondatori di città o inventori delle "arti" o eroi o astri o demoni. E questo - nota l'autore - risulta evidente soprattutto nei Platonici, che più degli altri si avvicinarono alla vera concezione della divinità. ${ }^{33}$

La critica agli "antiqui philosophantes" (la formula isidoriana "philosophi gentium" non viene mai usata) è tratta naturalmente da Agostino, alla cui testimonianza l'autore della Summa philosophiae si rifà più volte. Tale critica, ripresa a

12. Summa philosophiae Roberto Grosseteste ascripta, 1, 6, in L.BAUR, Die philosophischen Werke des Robert Grosseteste, Bischofs von Lincoln, Münster, Aschendorff, 1912 (BGPhM, 9), pp. 279-280.

13 Ibi, p. 280. 
distanza di parecchi secoli, si traduce però in una vera e propria periodizzazione, imperniata sui "tempora Theodosii magni". La scelta di quest'epoca quale spartiacque fra i due periodi va probabilmente collegata all'editto di Tessalonica, che operó una cesura rispetto al paganesimo e alla sua filosofia, segnando la definitiva affermazione del cristianesimo. Ma è soprattutto interessante rilevare come questa divisione in due periodi, di cui il secondo è superiore al primo, vada oltre l'originario ambito agostiniano della discussione sulla teologia naturale e sulla superiorità della religione cristiana, investendo anche quello studio della natura cui il retore Agostino era stato poco incline e che occupava invece un posto preminente nella scuola di Oxford, da cui proviene la Summa philosophiae. Difatti nella seconda parte di questo capitolo l'autore non si limita ad accennare - sulla scorta del De civitate Dei. - alle dottrine teologiche di Ermete Trismegisto, Platone e Porfirio, e ai numerosi errori che $i$ filosofi antichi nutrirono intorno all'anima, alla retribuzione finale e all'al di là. Egli si sofferma pure a denunciare l'insufficiente conoscenza che gli antichi ebbero, rispetto ai 'moderni', nel campo dell'astro-nomia e della filosofia naturale, e da tale giudizio negativo sono esclusi solo i "peripatetici" e Boezio, maestro nelle discipline del quadrivium: il termine di paragone è rappresentato, evidentemente, dalla scienza 'moderna' che gli Arabi hanno da poco trasmesso all'occidente latino e di cui la Summa philosophiae intende offrire un compendio."

Senso della continuità con il passato, dunque, ma anche consapevolezza di un novum che i 'moderni' a buon diritto possono rivendicare: con un pizzico di enfasi potremmo dire che è proprio questo lo spirito che anima il pensiero del XIII secolo. Ed ecco allora che, accanto alle grandi sistemazioni scolastiche, si avverte anche l'interesse per una rilettura globale del corso storico dell'umana sapienza, con significative varianti nella scansione o periodizzazione di tale corso. Nel suo Opus maius, ad es., Ruggero Bacone fuse insieme le prospettive della storia sacra e profana: all'età più antica, in cui i patriarchi biblici misero a frutto la loro eccezionale longevità per sviluppare lo studio della sapienza che in loro era stata infusa da Dio, segui un periodo di decadenza dovuta alla "malizia", che aveva allontanato gli uomini dal culto del vero Dio. La filosofia risorse con Salomone, ma poi tornò nuovamente a decadere per i peccati degli uomini, sino a che non venne ripresa da Talete, approfondita dai successori, portata al più alto livello da Aristotele, anche se è impossibile raggiungere la vera perfezione, perché nulla vi è di perfetto nelle umane scoperte. ${ }^{\text {tb }}$ Aristotele fu grande, ma - rileva Bacone - coloro che sono venuti dopo di lui hanno corretto ed arricchito le sue dottrine. Dopo un periodo di eclissi la filosofia di Aristotele riprese vigore con gli Arabi; ci aspetteremmo, a questo punto, un apprezzamento dell' "ingresso" del corpus aristotelico nell'occidente latino: invece Bacone è assai critico verso i contemporanei, che trascurano la logica nova, nonché le scienze matematiche e naturali e l'etica, rifugiandosi nello studio del diritto canonico... ${ }^{16}$

14 Ivi, p. 281.

15 The "Opus majus" of Roger Bacon, ed. J.H.Bridges, Oxford, Clarendon Press, 1897-1900 (rist. Frankfurt a.M., Minerva G.m.b.H., 1964), I, pp. 64-65.

16

Ibi, pp. 31 e $55-56$. 
Contemporaneo di Ruggero Bacone, e pure lui francescano (e inglese) era Giovanni di Galles, autore di un Compendiloquium de vita et dictis illustrium philosophorum. Nella parte conclusiva, dopo aver ampiamente illustrato la tradizionale divisione dei filosofi in sètte, Giovanni traccia una sorta di 'geografia' della storia filosofica e culturale, ispirandosi a quel tema della translatio studii che era stato toccato anche nel Chronicon di Ottone di Frisinga: sorta in oriente, la sapienza si trasferi dapprima in Grecia e poi a Roma, quindi a Parigi, al tempo di Carlo Magno, e infine ad Oxford... Giovanni di Galles appare qui animato da un'esigenza di "storicità", che egli soddisfa ricorrendo ad una categoria storica (la translatio studii, corrispettivo culturale della translatio imperii) che risulta intermedia fra l'onnicomprensivo schema agostiniano delle sei età del mondo e gli schemi biodossografici e diadochistici ereditati dall'antichità, nei quali la dimensione temporale è appiattita e priva di senso."

Ma è tempo di trarre alcune conclusioni da questa scorribanda attraverso l'età medievale. Una prima osservazione riguarda l'effetto ambivalente del "ritorno" di Aristotele nella cultura latino-medievale. In un primo tempo esso segnò l'ampliarsi della nozione di storicità della filosofia, non più racchiusa entro l'agostiniana prospettiva dei "Platonici" o il diffidente atteggiamento di Isidoro di Siviglia, che vedeva nei "philosophi gentium" i promotori di eresie. ${ }^{18}$ Tuttavia a questo dilatarsi della dimensione storica si accompagna ben presto la cristallizzazione della figura del 'filosofo' secondo schemi e moduli puramente logicospeculativi. Aristotele diviene un modello che, una volta affermatosi per il suo rigore dimostrativo e il suo carattere enciclopedico, fagocita le altre figure di filosofi, assurgendo ad espressione della ragione tout court più che della diuturna $\mathrm{e}$ contrastata ricerca della verità da parte di una multiforme "filosofica famiglia"; ma in tal modo è lo stesso circuito storico, che pure la riscoperta di Aristotele aveva arricchito e vitalizzato, a perdere valore di fronte all'esaltante progetto di un sapere rigoroso, fondato sul metodo sillogistico-deduttivo ed esteso a tutti i campi, dalla filosofia della natura alla stessa teologia. Ed è nella teologia che si coglie in particolare l'"effetto Aristotele": da esegesi della sacra pagina e da lectio historiae essa tende a trasformarsi in una "scienza", fondata non più sull'ordine temporale ma su quello logico, di per sé indifferente ai tempi e ai luoghi. Significativamente, l'attenuarsi della prospettiva storica (ed escatologica) nella nuova teologia elaborata dalla scolastica fa sì che tale prospettiva diventi patrimonio pressoché esclusivo dei movimenti profetici ed ereticali, che coltivano il senso della speranza e dell'attesa quale chiave di lettura globale della storia umana.

Questa sorta di mutazione culturale che caratterizza il movimento scolastico nel suo complesso si riflette anche nel modo d'intendere la storia della sapienza e dei filosofi. Non a caso nel tardo medioevo il tema "de vita et dictis philosophorum" sembra di pertinenza più storico-letteraria che strettamente filosofica, segno di un distacco fra il piano della "verità" e quello della "storia", fra l'epistème e

17 Florilegium de vita et dictis illustrium philosophorum et breviloquium de sapientia sanctorum, auctore IOANNE GUALLENSI Ordinis Minorum. Recensuit, et nunc primum edidit Fr. LUCAS WADDINGUS [...], Romae, apud N.A.Tinassium, 1655, pp. 409-424.

18 Cfr. ISID., Etym. 8, 6, 23 
l'istoria: un distacco inammissibile alla luce della teologia agostiniana della storia e della cultura. E non a caso la moderna storiografia filosofica, intesa come storia 'generale' dell'umana sapienza, si sviluppa nel XVII secolo non nei paesi cattolici in cui s'era affermata la seconda scolastica, bensì nei paesi di cultura protestante, dove la Riforma aveva ripreso e attualizzato il concetto di storia universale di matrice agostiniana. ${ }^{19} \mathrm{E}$ in tale prospettiva che si può meglio cogliere - e qui ci ricolleghiamo alle ben note tesi di Karl Löwith - la genesi della 'teologia laica' della storia (e della storia della cultura) sottesa alle grandi ricostruzioni storiche di Condorcet e di Hegel, di Comte e di Marx, e alla copiosa produzione storico-filosofica da esse derivata in questi due ultimi secoli... Ma sviluppare questo tema richiederebbe ben altro spazio: mi limito pertanto a questi provocatori spunti di riflessione, nati da un'indagine che si presentava, in apparenza, meramente erudita.

${ }_{19}$ Cfr. in proposito la Storia delle storie generali della filosofia, a cura di G.Santinello, I: Dalle origini rinascimentali alla "historia philosophica"; II: Dall'età cartesiana a Brucker, Brescia, La Scuola, 1979-1981. 